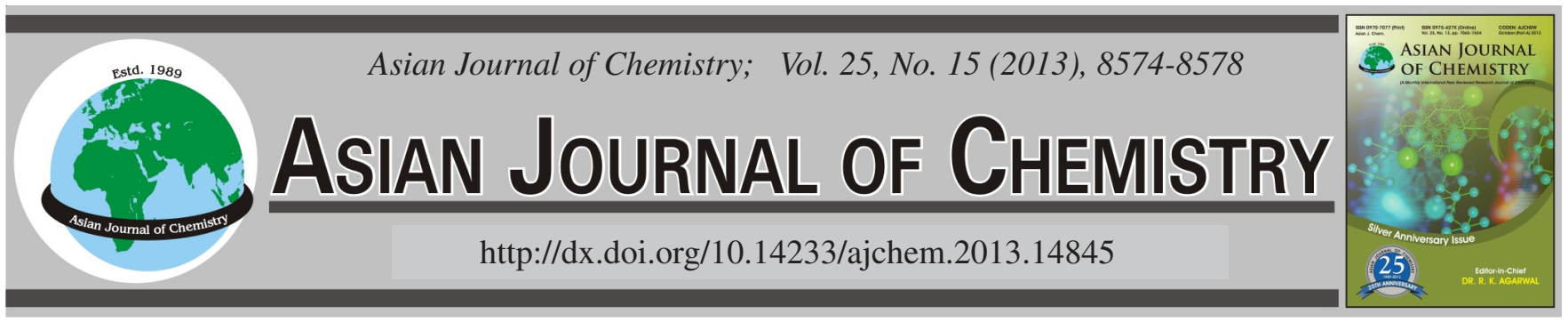

\title{
Synthesis and DFT-Based Quantitative Structure-Activity Relationships Study for Diphenyl Ethers Bactericide
}

\author{
J. YANG, W.Q. YANG, Y.Y. ZhANG, Y. MA and M.L. MA*
}

Key Lab of Advanced Scientific Computation of Sichuan province, Faculty of physical and Chemistry, Xihua University, Chengdu 610039, P.R. China

*Corresponding author: Fax: +8628 87723006; E-mail: mmlchem@163.com

(Received: 21 November 2012;

Accepted: 26 August 2013)

AJC-13999

Density functional theory (DFT) and linear regression analysis method are used to investigate the quantitative structure-activity relationship
(QSAR) of diphenyl ethers bactericide. It is found that there are good linear relationships between the experimental biological activity
data $[-\log (1 / \mathrm{MIC})]$ and the calculated energy gap of highest occupied molecular orbital and lowest unoccupied frontier orbital $\left(\Delta \mathrm{E}_{\mathrm{gap}}\right)$.
The $2 \mathrm{D}-\mathrm{QSAR}$ equations $\left(\mathrm{R}^{2}=0.87942\right)$ was established used $-\log (1 / \mathrm{MIC})$ and $\Delta \mathrm{E}_{\text {gap }}$ as factors. A validation set of 14 diphenyl ethers
were selected and their activities were computed using the proposed QSAR model and three of them were synthesized and characterized.
The correlation between the predicted and observed activities was excellent.
Key Words: Biological activities, Diphenyl ethers, QSAR, DFT, Synthesis.

\section{INTRODUCTION}

A kind of widely used organic compounds, halogenated hydroxy-diphenyl ethers possess good antibacterial activity and are applied in many fields as bactericide and germicide. A series of hydroxy diphenyl ethers compound were designed and synthesized by our research group via three different routes $\left(\right.$ Scheme-I) ${ }^{1-5}$.

There are no report is available in literature before structure designed and synthesis work for the relationship between the structure and biological activity. Structure-activity relationship (QSAR) analysis show wide applications due to their well established predictive power ${ }^{6}$. Essentially, correlating the physico-chemical properties of a series of compounds with their respective biological activities is believed to provide a useful tool in designing new drugs $\mathrm{s}^{7,8}$.
In order to explore the relationship between structure and antibacterial activity of diphenyl ethers, density functional theory (DFT) and linear regression analysis method were used to construct the QSAR models of diphenyl ethers. Used the QSAR equation, the biological activity of new diphenyl ethers bactericide can be predicted. The predicted results provided an important reference for the future research work of designing and synthesis new derivative.

\section{EXPERIMENTAL}

Thin layer chromatography was performed with Qingdao Ocean silica gel $\mathrm{GF}_{254}$ and flash column chromatography was carried out with Qingdao Ocean gel 100-200 mes. Nuclear magnetic resonance (nmr) spectra were recorded on a Bruker DPX-300 FT NMR spectrometer at $300 \mathrm{MHz}$ for ${ }^{1} \mathrm{H}$ and were referenced to tetramethylsilane ( $\delta$ values are given in ppm and

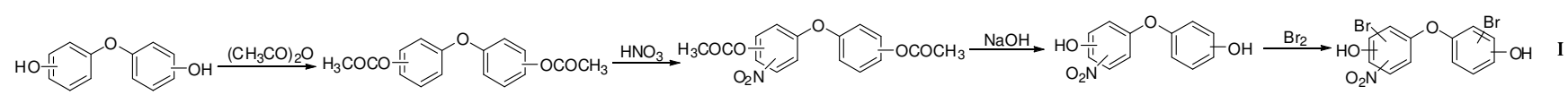

管

等

Scheme-I: Synthesis route of the diphenyl ethers compound 
$J$-values in $\mathrm{Hz}$ ). Mass spectra were measured on a Varian $\mathrm{CH}-5$ apparatus. IR spectra were measured on Nicolet380 and obtained using $\mathrm{KBr}$ plates.

4-(3-Bromo-5-hydroxyphenoxy)-3-nitrophenol ( $\left.\mathbf{a}_{7}\right)$ : Potassium hydroxide $(55 \mathrm{mmol})$ was added to a solution of the 1-chloro-4-methoxy-2-nitrobenzene $(60 \mathrm{mmol})$ in DMF $(65 \mathrm{~mL})$ at room temperature. The mixture was stirred for $0.5 \mathrm{~h}$ and then $50 \mathrm{mmol}$ of 5-bromobenzene-1,3-diol was added. The mixture was heated at reflux for $1 \mathrm{~h}$ under nitrogen. The cooled mixture was poured onto $250 \mathrm{~mL}$ water and the precipitate was collected by filtration, washed twice with $5 \%$ aqueous $\mathrm{NaOH}$ and twice with $\mathrm{H}_{2} \mathrm{O}$ to give crude 3-bromo-5(4-methoxy-2-nitrophenoxy)phenol. The 3-bromo-5-(4methoxy-2-nitrophenoxy)phenol and $80 \mathrm{~mL}$ of $48 \% \mathrm{HBr}$ were stirred in refluxing at $125^{\circ} \mathrm{C}$ for $24 \mathrm{~h}$. After cooling, the mixture was neutralized with sodium hydroxide and was extracted several times with MTBE. The combined MTBE extract was washed twice with $\mathrm{H}_{2} \mathrm{O}$ and dried over $\mathrm{MgSO}_{4}$, filtration and evaporation of the solvent. The residue was subjected to column chromatography on silica gel (100-200 mesh) eluted with $35 \% \mathrm{EtOA} / \mathrm{chexanes}$ to yield $\mathbf{a}_{7}$ as a yellow solid. Yield $29 \%,{ }^{1} \mathrm{H} \mathrm{NMR}\left(300 \mathrm{MHz}, \mathrm{CDCl}_{3}\right): 7.91(\mathrm{~d}, J=3.0 \mathrm{~Hz}, 1 \mathrm{H}$, ArH), 7.78 (dd, $J=9.0,3.0 \mathrm{~Hz}, 1 \mathrm{H}, \mathrm{ArH}), 7.30$ (dd, $J=9.0$, $3.0 \mathrm{~Hz}, 1 \mathrm{H}, \mathrm{ArH}), 7.13$ (d, $J=3.0 \mathrm{~Hz}, 1 \mathrm{H}, \mathrm{ArH}), 7.00$ (d, $J=$ $9.0 \mathrm{~Hz}, 1 \mathrm{H}, \mathrm{ArH}), 6.89$ (d, J = 9.0 Hz, 1H, ArH), 6.41 (s, 1H, $\mathrm{OH}), 5.92$ (s, 1H, OH), IR (KBr, $\left.v_{\max }, \mathrm{cm}^{-1}\right): 3427,1593,1562$, 1509, 1349, 1268, 1210, MS m/z: 326, 324.

2-(3-Bromo-5-hydroxyphenoxy)-3-nitrophenol $\left(a_{8}\right)$ : The above procedure was used to prepare $\mathbf{a}_{8}$ from 2-chloro-1methoxy-3-nitrobenzene and 5-bromobenzene-1,3-diol. Yield $23 \%,{ }^{1} \mathrm{H}$ NMR $\left(\mathrm{CDCl}_{3}, 300 \mathrm{MHz}\right) \delta: 7.92(\mathrm{~d}, J=3.0 \mathrm{~Hz}, 1 \mathrm{H}$, ArH), $7.76(\mathrm{dd}, J=9.0,3.0 \mathrm{~Hz}, 1 \mathrm{H}, \mathrm{ArH}), 7.28(\mathrm{~d}, J=2.8 \mathrm{~Hz}$, $1 \mathrm{H}, \mathrm{ArH}), 7.12$ (d, $J=9.0 \mathrm{~Hz}, 1 \mathrm{H}, \mathrm{ArH}), 7.04$ (dd, $J=9.0,2.8$ $\mathrm{Hz}, 1 \mathrm{H}, \mathrm{ArH}), 6.78$ (d, $J=9.0 \mathrm{~Hz}, 1 \mathrm{H}, \mathrm{ArH}), 5.92$ (s, 1H, $\mathrm{OH}), 5.51(\mathrm{~s}, 1 \mathrm{H}, \mathrm{OH}), \mathrm{IR}\left(\mathrm{KBr}, \mathrm{v}_{\max }, \mathrm{cm}^{-1}\right) \mathrm{v}: 3368,1602$, 1510, 1480, 1347, 1269, 1227, MS m/z: 326, 324.

2-(3-Bromo-5-hydroxyphenoxy)-6-nitrophenol ( $\left(\mathrm{a}_{15}\right)$ : The procedure above was used to prepare $\mathbf{a}_{15}$ from 1-chloro2-methoxy-3-nitrobenzene and 5-bromobenzene-1,3-diol. Yield $27 \%,{ }^{1} \mathrm{H}$ NMR $\left(\mathrm{CDCl}_{3}, 300 \mathrm{MHz}\right) \delta: 7.45$ (d, $J=2.8$ $\mathrm{Hz}, 1 \mathrm{H}, \mathrm{ArH}), 7.15$ (d, $J=2.8 \mathrm{~Hz}, 1 \mathrm{H}, \mathrm{ArH}), 7.09$ (dd, $J=$ 8.8, $2.8 \mathrm{~Hz}, 1 \mathrm{H}, \mathrm{ArH}), 7.06$ (d, $J=8.8 \mathrm{~Hz}, 1 \mathrm{H}, \mathrm{ArH}), 6.97$ (d, $J=8.8 \mathrm{~Hz}, 1 \mathrm{H}, \mathrm{ArH}), 6.86(\mathrm{dd}, J=8.8,2.8 \mathrm{~Hz}, 1 \mathrm{H}, \mathrm{ArH})$, $5.54(\mathrm{~s}, 1 \mathrm{H}, \mathrm{OH}), 5.34(\mathrm{~s}, 1 \mathrm{H}, \mathrm{OH}), \mathrm{IR}\left(\mathrm{KBr}, \mathrm{v}_{\max }, \mathrm{cm}^{-1}\right): 3385$, 1596, 1529, 1495, 1324, 1258, 1219, MS m/z: 326, 324.

The minimum inhibitory concentrations (MIC) of hydroxy diphenyl ethers were determined by the agar dilution method used Staphylococcus aureus as testing bacteria9. Hydroxy diphenyl ethers were dissolved in $75 \%$ of 2-methoxyethanol and agar plates were prepared that contained this biocide in dilutions ranging from $0-1024 \mu \mathrm{g} / \mathrm{mL}$. Plates were inoculated to be absorbed into agar before incubation. Conditions of incubation were $35-37^{\circ} \mathrm{C}$ in air for $20 \mathrm{~h}$. The MIC was defined as lowest concentration of antibiotic at which there is no visible growth of the organism.

\section{RESULTS AND DISCUSSION}

There are 24 diphenyl ethers (Scheme-II), which were synthesized by our research group, were used for constructing the 2D-QSAR models. The optimized molecular geometry of compound was computed first. All computations were carried out using the Gaussian $03^{10}$ computer software package. The electronic descriptors were obtained from a single-point calculation at the B3LYP/6-311+g (d) level. The optimized geometrical parameters are given in Table-1.

The frontier orbital theory states that the energy of the HOMO and LUMO are the important factors that determine the reactivity of a molecule. The chemicals which have greater ability to accept electron density through charge-transfer interaction should bind to the aryl hydrocarbon receptor with greater affinity than those with lower electron-acceptor properties $^{11,12}$. This would suggest that these stronger electron acceptors should have a lower energy unoccupied frontier orbital $\left(\mathrm{E}_{\mathrm{Lumo}}\right)$, lower energy for the occupied frontier orbital $\left(\mathrm{E}_{\text {номо }}\right)$ and a lower energy difference in these frontier orbitals $\left(\Delta \mathrm{E}_{\mathrm{gap}}\right)$, which can be related to molecular reactivity ${ }^{13,14}$. So the energy of geometry optimized diphenyl ethers was calculated and the $\mathrm{HF}, \mathrm{E}_{\mathrm{HOMO}}, \mathrm{E}_{\mathrm{LumO}}$ and $\Delta \mathrm{E}_{\text {gap }}$ Value were summarized in Table-2.

It was found that the MIC of diphenyl ethers compounds have a certain relation with theoretical data of the $\Delta \mathrm{E}_{\text {gap }}$, the antibacterial activity showed a downward trend with the increasing of $\Delta \mathrm{E}_{\text {gap }}$ values. The present study presents a comprehensive QSAR analysis for diphenyl ethers as a bactericide drug. A multiple regression analysis was carried out and we arrived at the final QSAR equation. Thus, the QSAR equation can be written as:

$$
-\log \left(\frac{1}{\mathrm{MIC}}\right)=-1.6868+20.9237 \times \Delta \mathrm{E}_{\mathrm{gap}}
$$

$\mathrm{n}=24, \mathrm{R}^{2}=0.877212, \mathrm{SD}=0.27871, \mathrm{~F}=45.081, \mathrm{P}<0.0001$.

The QSAR equations possess relatively high correlation coefficient R, low standard deviation SD and least number of<smiles></smiles><smiles>O=[N+]([O-])c1ccc(O)c(Oc2ccc(Br)cc2Br)c1</smiles><smiles>O=[N+]([O-])c1cccc(Oc2ccc(Br)cc2O)c1Br</smiles><smiles>Cc1ccc(Oc2ccc(F)cc2)c(O)c1</smiles><smiles>Oc1ccc(Oc2ccc(F)cc2)cc1Br</smiles><smiles>Cc1ccccc1Oc1ccc(O)c(Br)c1</smiles><smiles>Cc1ccc(Oc2ccc(CCc3ccc(Oc4cc(Br)ccc4O)cc3)c(Br)c2)cc1</smiles><smiles>Oc1ccc(Oc2ccc(Br)cc2)cc1Br</smiles><smiles>Cc1cc(Br)cc(Oc2ccc(O)c(O)c2)c1</smiles><smiles>Cc1ccccc1Oc1ccc(Br)cc1Oc1ccc(Br)c(O)c1O</smiles><smiles>Cc1ccc(Oc2ccc(Br)cc2O)c(Br)c1</smiles><smiles>O=[N+]([O-])c1ccc(Oc2cc(Br)ccc2O)cc1</smiles>

$d_{1}$ $\mathrm{d}_{2}$<smiles>Cc1cccc(Oc2ccc(Br)cc2Oc2ccc(O)c(O)c2)c1</smiles><smiles>O=[N+]([O-])c1ccc(Oc2ccc(O)c(Br)c2)cc1</smiles><smiles>Oc1ccc(Oc2ccc(Br)cc2)cc1</smiles><smiles>Oc1ccc(Oc2ccc(O)c(Br)c2)cc1</smiles>

Scheme-II: Structure of the diphenyl ethers compound used for constructing the QSAR models 


\begin{tabular}{|c|c|c|c|c|c|c|c|c|c|c|c|}
\hline Compound & $\begin{array}{c}\text { Ring A } \\
\text { C-N }\end{array}$ & $\begin{array}{c}\text { Ring A } \\
\text { C-O }\end{array}$ & $\begin{array}{c}\text { Ring A } \\
\text { C-F }\end{array}$ & $\begin{array}{l}\text { Ring A } \\
\mathrm{C}_{-} \mathrm{CH}_{3}\end{array}$ & $\begin{array}{c}\text { Ring A } \\
\text { C-Br }\end{array}$ & $\begin{array}{l}\text { Ring A- } \\
\mathrm{O}\end{array}$ & $\begin{array}{c}\text { O-Ring } \\
\text { B }\end{array}$ & $\begin{array}{c}\text { Ring B } \\
\text { C-O }\end{array}$ & $\begin{array}{l}\text { Ring B } \\
\text { C-Br }\end{array}$ & $\begin{array}{c}\text { Ring B } \\
\text { C-Br }\end{array}$ & $\begin{array}{l}\text { Dihedral } \\
\text { angle }\end{array}$ \\
\hline a1 & 1.47444 & 1.35859 & - & - & - & 1.36958 & 1.37974 & 1.36253 & 1.91772 & - & 28.20258 \\
\hline $\mathbf{a} 2$ & 1.47317 & 1.35727 & - & - & - & 1.37674 & 1.39386 & 1.35433 & 1.91854 & - & 69.08806 \\
\hline a3 & 1.47615 & 1.36382 & - & - & - & 1.36882 & 1.38846 & 1.35863 & 1.92239 & - & 27.14498 \\
\hline a4 & 1.47850 & 1.35656 & - & - & - & 1.36872 & 1.38455 & 1.36361 & 1.91602 & - & 33.30459 \\
\hline a5 & 1.44190 & 1.33620 & - & - & - & 1.36400 & 1.39117 & 1.36224 & 1.91365 & - & 80.35678 \\
\hline b3 & 1.47553 & 1.36308 & - & - & - & 1.37219 & 1.38283 & 1.35193 & 1.92049 & 1.90653 & 25.23897 \\
\hline b1 & 1.47531 & 1.35843 & - & - & - & 1.37154 & 1.37677 & 1.35097 & 1.91927 & 1.91505 & 27.33849 \\
\hline b2 & 1.47437 & 1.35707 & - & - & - & 1.37928 & 1.39116 & 1.34770 & 1.91806 & 1.90338 & 70.88311 \\
\hline b4 & 1.46776 & 1.35041 & - & - & - & 1.38618 & 1.38319 & 1.35044 & 1.91126 & 1.91660 & 71.56889 \\
\hline b5 & 1.45628 & 1.33326 & - & - & - & 1.37538 & 1.37562 & 1.35120 & 1.91946 & 1.91530 & 22.26875 \\
\hline c1 & - & - & 1.35880 & - & - & 1.38470 & 1.37686 & 1.36196 & 1.91661 & - & 73.18478 \\
\hline c2 & - & - & 1.35774 & - & - & 1.38171 & 1.38441 & 1.36218 & 1.90741 & - & 57.98806 \\
\hline c3 & - & - & 1.35004 & - & - & 1.37521 & 1.37831 & 1.36141 & 1.91638 & - & 18.90108 \\
\hline d1 & - & - & - & 1.50586 & - & 1.38882 & 1.37505 & 1.36224 & 1.91732 & - & 18.86771 \\
\hline d2 & - & - & - & 1.50599 & - & 1.38764 & 1.38254 & 1.36235 & 1.90896 & - & 21.92345 \\
\hline d3 & - & - & - & 1.50639 & - & 1.38562 & 1.38260 & 1.36269 & 1.90888 & - & 22.62333 \\
\hline d4 & - & - & - & 1.50968 & - & 1.38409 & 1.38231 & 1.36318 & 1.90827 & - & 29.94973 \\
\hline d5 & - & - & - & 1.50993 & - & 1.38596 & 1.37554 & 1.36186 & 1.91755 & - & 16.29069 \\
\hline e1 & - & - & - & - & 1.91897 & 1.38116 & 1.37819 & 1.36159 & 1.91640 & - & 110.16884 \\
\hline e2 & - & - & - & - & 1.91832 & 1.37825 & 1.38700 & 1.36122 & 1.90708 & - & 71.99985 \\
\hline f1 & 1.46887 & - & - & - & & 1.36953 & 1.38323 & 1.36110 & 1.91466 & - & 77.43238 \\
\hline f2 & 1.46894 & - & - & - & & 1.36716 & 1.39261 & 1.35933 & 1.90554 & - & 96.36661 \\
\hline g1 & - & 1.36475 & - & - & 1.91988 & 1.36941 & 1.39113 & 1.36907 & - & - & 95.96239 \\
\hline g2 & - & 1.36093 & - & - & 1.92445 & 1.37936 & 1.38932 & 1.36929 & - & - & 14.20552 \\
\hline
\end{tabular}

TABLE-2

CALCULATED ENERGY VALUE AND THE OBSERVED ACTIVE VALUES OF DIPHENYL ETHERS

\begin{tabular}{|c|c|c|c|c|c|c|c|c|}
\hline Structure & Compound & $\begin{array}{c}\text { HF } \\
\text { (Hartree) }\end{array}$ & $\begin{array}{c}\mathrm{E}_{\text {Номо }} \\
\text { (Hartree) }\end{array}$ & $\begin{array}{c}\mathrm{E}_{\text {LUMO }} \\
\text { (Hartree) }\end{array}$ & $\begin{array}{c}\Delta \mathrm{E}_{\text {gap }} \\
\text { (Hartree) }\end{array}$ & $\begin{array}{c}\Delta \mathrm{E}_{\mathrm{gap}} \\
(\mathrm{kJ} / \mathrm{mol})\end{array}$ & $\begin{array}{c}\mathrm{MIC} \\
(\mu \mathrm{g} / \mathrm{mL})\end{array}$ & $-\log (1 / \mathrm{MIC})$ \\
\hline \multirow{5}{*}{$\mathrm{O}_{2} \mathrm{~N}$} & $\mathbf{a}_{3}$ & -3467.2193396 & -0.23103 & -0.10894 & 0.12209 & 320.547295 & 8 & 0.90309 \\
\hline & $\mathbf{a}_{1}$ & -3467.2309972 & -0.23548 & -0.10855 & 0.12693 & 333.254715 & 12 & 1.07918 \\
\hline & $\mathbf{a}_{2}$ & -3467.2155827 & -0.25149 & -0.10095 & 0.15054 & 395.242770 & 16 & 1.20412 \\
\hline & $\mathbf{a}_{5}$ & -3467.2377244 & -0.26035 & -0.10952 & 0.15083 & 396.004165 & 24 & 1.38021 \\
\hline & $\mathbf{a}_{4}$ & -3467.2129707 & -0.24809 & -0.08627 & 0.16182 & 424.858410 & 64 & 1.80618 \\
\hline \multirow{5}{*}{ (N) } & $\mathbf{b}_{5}$ & -6040.7557998 & -0.23975 & -0.12345 & 0.11630 & 305.34570 & 2 & 0.30103 \\
\hline & $\mathbf{b}_{3}$ & -6040.7633589 & -0.23793 & -0.10724 & 0.13069 & 343.126595 & 20 & 1.30103 \\
\hline & $\mathbf{b}_{1}$ & -6040.7691917 & -0.24294 & -0.11171 & 0.13123 & 344.544365 & 20 & 1.30103 \\
\hline & $\mathbf{b}_{2}$ & -6040.7722341 & -0.25574 & -0.10269 & 0.15305 & 401.832775 & 64 & 1.80618 \\
\hline & $\mathbf{b}_{4}$ & -6040.7688792 & -0.25542 & -0.10019 & 0.15523 & 407.711595 & 128 & 2.10721 \\
\hline \multirow{3}{*}{$\pi^{0} \pi_{B=}^{O}$} & $\mathbf{c}_{1}$ & -3286.6855684 & -0.04206 & -0.23500 & 0.19294 & 506.56397 & 200 & 2.30103 \\
\hline & $c_{2}$ & -3286.6853119 & -0.04616 & -0.23120 & 0.18504 & 485.82252 & 128 & 2.10721 \\
\hline & $c_{3}$ & -3286.6815839 & -0.04069 & -0.23699 & 0.19630 & 515.38565 & 280 & 2.44716 \\
\hline \multirow{4}{*}{$\mathrm{H}_{3} \mathrm{C}+\mathrm{C}$} & $d_{2}$ & -3226.7408792 & -0.03080 & -0.22339 & 0.19259 & 505.645045 & 386 & 2.58659 \\
\hline & $d_{3}$ & -3226.7422464 & -0.03962 & -0.22671 & 0.18709 & 491.204795 & 380 & 2.57978 \\
\hline & $d_{4}$ & -3226.7413883 & -0.03908 & -0.22323 & 0.18415 & 483.485825 & 360 & 2.55630 \\
\hline & $d_{5}$ & -3226.7420546 & -0.03687 & -0.23010 & 0.19323 & 507.325365 & 400 & 2.60206 \\
\hline \multirow{2}{*}{$\mathrm{Br} \frac{\pi}{11} y^{0}$} & $\mathbf{e}_{1}$ & -5760.960438 & -0.04380 & -0.23462 & 0.19082 & 500.99791 & 124 & 2.09342 \\
\hline & $\mathbf{e}_{2}$ & -5760.9601324 & -0.04776 & -0.23444 & 0.18668 & 490.12834 & 88 & 1.94448 \\
\hline \multirow{4}{*}{$\mathrm{O}_{2} \mathrm{~N} \mathbb{1}$} & $\mathbf{f}_{1}$ & -3391.9840696 & -0.09870 & -0.25140 & 0.15270 & 400.91385 & 12 & 1.07918 \\
\hline & $\mathbf{f}_{2}$ & -3391.9835342 & -0.10039 & -0.25407 & 0.15368 & 403.48684 & 24 & 1.38021 \\
\hline & $\mathbf{g}_{1}$ & -3262.6567364 & -0.03386 & -0.22421 & 0.19035 & 499.763925 & 48 & 1.68124 \\
\hline & $\mathbf{g}_{2}$ & -3262.6618251 & -0.03918 & -0.22181 & 0.18263 & 479.495065 & 42 & 1.62325 \\
\hline
\end{tabular}

variables. The high values of $\mathrm{E}_{\text {номо }}$ are likely to indicate a tendency of the molecule to donate electrons to appropriate acceptors and the lower value of $\mathrm{E}_{\mathrm{LUMO}}$, the more probable, it is the molecule would accept electrons ${ }^{15}$. So the values of the $\Delta \mathrm{E}_{\text {gap }}$ show that higher efficiency can be related to a lower energy difference. $\mathrm{A}$ large $\Delta \mathrm{E}_{\text {gap }}$ implies high stability for the molecule in the sense of its lower sensitivity in the biochemical processes.

Especially in the same series, where their have same substituent but different substitution position, a better linear 


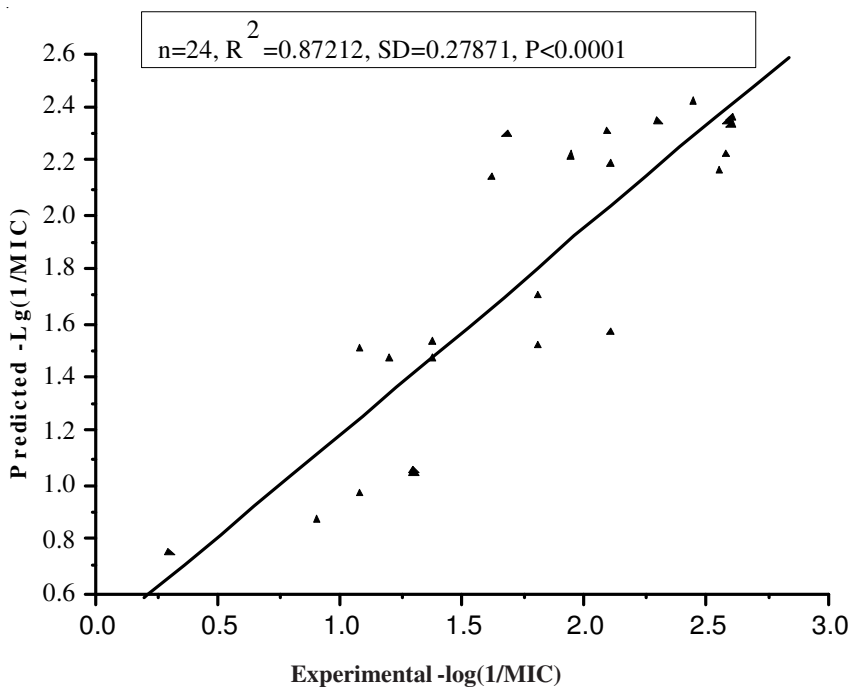

Fig. 1. Relationships between the $-\log (1 / \mathrm{MIC})$ values from experiment and prediction based on QSAR equations

relationships were found (Figs. 1 and 2), such as $\mathbf{a}_{1}-\mathbf{a}_{5}$ the $\mathrm{R}^{2}$ $=0.90115$. The same regularity also showed in the series " $b$ " $\left(\mathrm{R}^{2}=0.95704\right)$ and "d" $\left(\mathrm{R}^{2}=0.91897\right)$.

The QSAR models also show that the descriptors derived from DFT and linear regression analysis method can successfully be utilized to predict the antibacterial activity of the diphenyl ethers. A series of new diphenyl ethers $\mathbf{a}_{\mathbf{6}}-\mathbf{a}_{\mathbf{1 9}}$, which have same substituent but different substitution position, were designed and their structure showed in Scheme-III.

The geometry optimizations and energy calculations of new designed diphenyl ethers was done first. Used the QSAR equation, the biological activity of new diphenyl ethers bactericide were predicted and the result shown in Table- 3 . The calculated results show that $\mathbf{a}_{7}, \mathbf{a}_{6}$ and $\mathbf{a}_{15}$ (Table- 3 entry 2, 3 and 10) have lower MIC, so they were chosen as a target for the research work of synthesis and antibacterial activity test. Dihydroxy nitro diphenyl ethers were prepared by williamson reaction and then demethoxylation by $\mathrm{HBr}$ to give three new diphenyl ethers (Scheme-IV).

The structures of the new targets were confirmed by ${ }^{1} \mathrm{H}$ NMR, IR and MS spectra. Their biological activity was tested by the agar dilution method also. To our interest, the MIC data of the $\mathbf{a}_{7}, \mathbf{a}_{6}$ and $\mathbf{a}_{15}$ are similar to predictive value (Table3 entry 2, 3 and 10). Almost similar data of predicted and experimental biological activity fully proved the reliability and correctness of 2D-QASR equation. The antibacterial activities of the diphenyl ethers are highly dependent on the $\Delta \mathrm{E}_{\text {gap }}$ of the drug as measured by the electronic descriptor, i.e., $\Delta \mathrm{E}_{\text {gap }}$ is an important stability index.
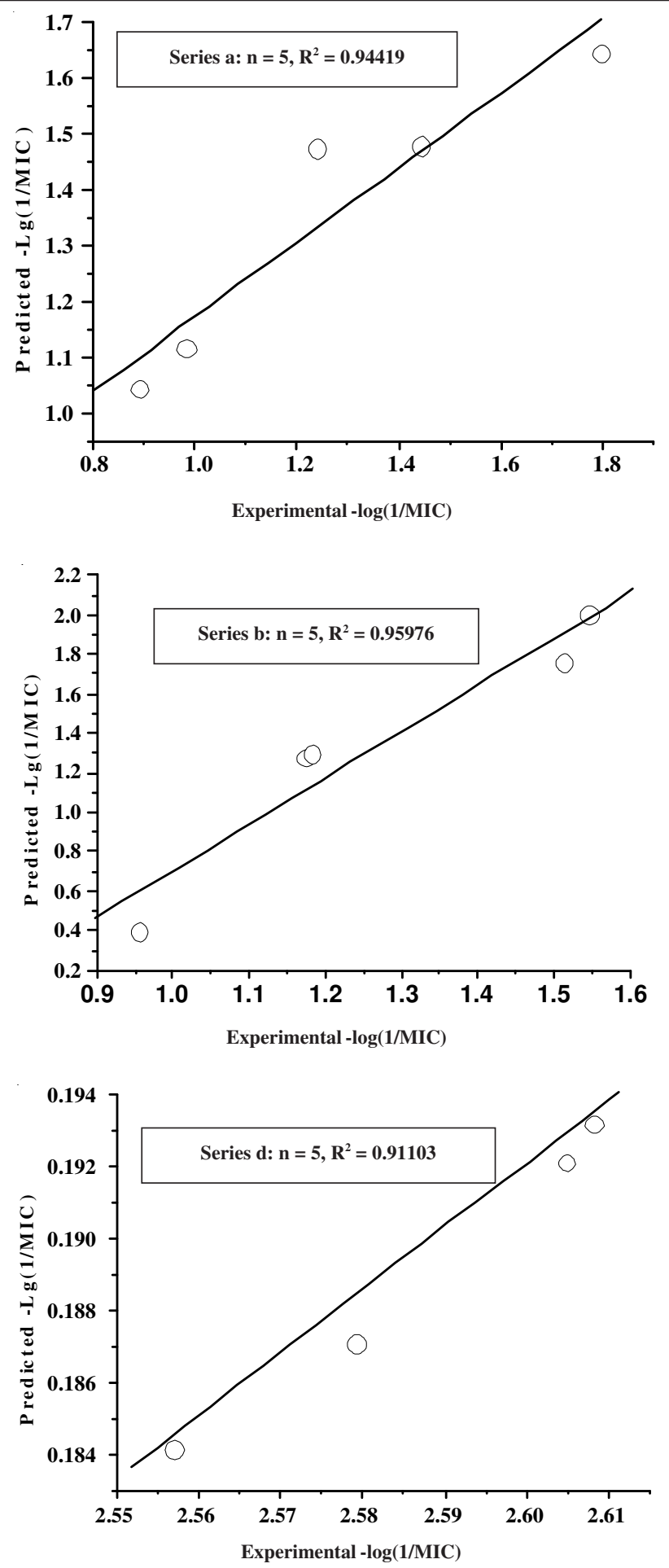

Fig. 2. Relationships between the $-\log (1 / \mathrm{MIC})$ from experiment and prediction in same series based on QSAR equations<smiles>O=[N+]([O-])c1ccc(Oc2cc(O)cc(Br)c2)c([N+](=O)[O-])c1</smiles>

$a_{6}$<smiles>O=[N+]=Nc1c([N+](=O)[O-])ccc(Oc2ccc(Br)cc2O)c1O</smiles><smiles>O=[N+]([O-])c1cccc(Oc2cc(O)cc(Br)c2)c1O</smiles><smiles>O=[N+]([O-])c1ccc(Oc2ccc(Br)cc2O)c(O)c1</smiles><smiles>O=[N+]([O-])c1ccc(O)cc1Oc1ccc(Br)cc1O</smiles><smiles>O=[N+]([O-])c1ccc(Oc2c(O)cccc2Br)c(O)c1</smiles><smiles>O=[N+]([O-])c1ccc(O)c(O)c1Oc1ccccc1Br</smiles><smiles>O=[N+]([O-])c1ccc(Oc2ccc(Br)cc2O)cc1O</smiles><smiles>O=[N+]([O-])c1ccc(Oc2ccccc2O)c(O)c1</smiles>

$a_{11}$

$a_{12}$<smiles>O=[N+]([O-])c1cccc(O)c1Oc1ccc(Br)cc1O</smiles><smiles>O=[N+]([O-])c1cccc(O)c1Oc1bcccc1O</smiles>

Scheme-III: Nnew designed diphenyl ethers compound 
TABLE-3

CALCULATED ENERGY AND PREDICTED ANTIBACTERIAL ACTIVITY OF NEW DIPHENYL ETHERS

\begin{tabular}{|c|c|c|c|c|c|c|c|}
\hline Compound & $\begin{array}{c}\text { HF } \\
\text { (Hartree) }\end{array}$ & $\begin{array}{c}\mathrm{E}_{\text {Номо }} \\
\text { (Hartree) }\end{array}$ & $\begin{array}{c}\mathrm{E}_{\text {LUMo }} \\
\text { (Hartree) }\end{array}$ & $\begin{array}{c}\Delta \mathrm{E}_{\text {gap }} \\
\text { (Hartree) }\end{array}$ & $\begin{array}{c}\text { Predicted activity } \\
\text { - } \log (1 / \text { MIC })\end{array}$ & $\begin{array}{c}\text { Predicted MIC } \\
(\mu \mathrm{g} / \mathrm{mL})\end{array}$ & $\begin{array}{c}\text { Expt. MIC } \\
(\mu \mathrm{g} / \mathrm{mL})\end{array}$ \\
\hline$a_{6}$ & -3467.2300951 & -0.10328 & -0.25532 & 0.15204 & 1.4831 & 30 & - \\
\hline $\mathbf{a}_{7}$ & -3467.2168032 & -0.10557 & -0.24608 & 0.14051 & 1.2417 & 17 & 16 \\
\hline $\mathbf{a}_{8}$ & -3467.2141501 & -0.10147 & -0.24109 & 0.13962 & 1.2231 & 17 & 16 \\
\hline $\mathbf{a}_{9}$ & -3467.2277055 & -0.09814 & -0.25054 & 0.15240 & 1.4906 & 31 & - \\
\hline$a_{10}$ & -3467.2252824 & -0.09588 & -0.24842 & 0.15254 & 1.4936 & 31 & - \\
\hline$a_{11}$ & -3467.2351969 & -0.10731 & -0.25602 & 0.14871 & 1.4134 & 26 & - \\
\hline$a_{12}$ & -3467.2317537 & -0.10623 & -0.2593 & 0.15307 & 1.5047 & 32 & - \\
\hline$a_{13}$ & -3467.2343426 & -0.10829 & -0.25624 & 0.14795 & 1.3975 & 25 & - \\
\hline $\mathbf{a}_{14}$ & -3467.2325464 & -0.10533 & -0.25754 & 0.15221 & 1.4867 & 31 & - \\
\hline$a_{15}$ & -3467.2303465 & -0.12215 & -0.24557 & 0.12342 & 0.8839 & 8 & 8 \\
\hline$a_{16}$ & -3467.2170951 & -0.08789 & -0.24965 & 0.16176 & 1.6866 & 49 & - \\
\hline$a_{17}$ & -3467.2138073 & -0.08533 & -0.2525 & 0.16717 & 1.7999 & 63 & - \\
\hline$a_{18}$ & -3467.2078235 & -0.09234 & -0.23917 & 0.14683 & 1.3740 & 24 & - \\
\hline$a_{19}$ & -3467.2045143 & -0.09193 & -0.23835 & 0.14642 & 1.3654 & 23 & - \\
\hline
\end{tabular}

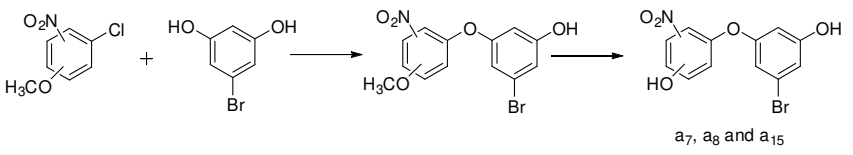

Scheme-IV: Synthesis route of the new diphenyl ethers compound

\section{Conclusion}

In summary, the quantitative structure-activity relationships of diphenyl ethers derivatives have been studied. The quantum chemical parameters of diphenyl ethers were calculated at the B3LYP/6- 311G** level, based on which the QSAR model of $-\log (1 / \mathrm{MIC})$ was proposed. The QSAR equation have better stability ability judging from the values of $\mathrm{R}^{2}(0.877212)$, $\mathrm{SD}(0.27871), p<0.00010$ and F-value (45.081). Base on QSAR equation, the biological activity of series new designed diphenyl ethers were predicted. Three new diphenyl ethers, which were calculated to have better biological activity, were synthesized and characterized. The biological activity test results further confirm the reliability and good predictive ability of QSAR model.

\section{ACKNOWLEDGEMENTS}

This work was supported by the NSFC (No. B020601), the NSF of Sichuan (No. 07ZA109) and the foundation of Xihua Univsrsity (No. XZD0912-09). The authors thank the Dr. Wang and his students of college of bioengineering of Xihua University for the test work of minimum inhibitory concentration (MIC).

\section{REFERENCES}

1. M.L. Ma, Y. Xing and Y.L. Wang, Chin. J. Org. Chem., 24, 927 (2004).

2. S. Cheng, Y. Xing and Y.L. Wang, Chin. J. Org. Chem., 28, 498 (2008).

3. Y. Zou, K.Q.Wu and Y.L. Wang, Chin. J. Org. Chem., 28, 111 (2008).

4. Y.L. Wang, M.L. Ma and R. Fu, Chin. J. Org. Chem., 24, 734 (2004).

5. M.L. Ma, Y. Xing and Y.L.Wang, J. Sichuan Univ. (Nat. Sci. Ed.), 01, 137 (2004).

6. C. Hansch, Acc. Chem. Res., 2, 232 (1969).

7. R. Hilal and A.K. Elroby, Mol. Simulation, 37, 62 (2011).

8. C.G. Gu, X.H. Ju and X. Sun, Ecotoxicol. Environ. Safety, 73, 1470 (2010).

9. J.M. Andrews, J. Antimicrobiol. Chemother, 48, 5 (2001).

10. M.J. Frisch, G.W. Trucks, H.B. Schlegel, G.E. Scuseria, M.A. Robb, J.R. Cheeseman, J.A. Montgomery Jr., T. Vreven, K.N. Kudin, J.C. Burant, J.M. Millam, S.S. Iyengar, J. Tomasi, V. Barone, B. Mennucci, M. Cossi, G. Scalmani, N. Rega, G.A. Petersson, H. Nakatsuji, M. Hada, M. Ehara, K. Toyota, R. Fukuda, J. Hasegawa, M. Ishida, T. Nakajima, Y. Honda, O. Kitao, H. Nakai, M. Klene, X. Li, J.E. Knox, H.P. Hratchian, J.B. Cross, C. Adamo, J. Jaramillo, R. Gomperts, R.E. Stratmann, O. Yazyev, A.J. Austin, R. Cammi, C. Pomelli, J.W. Ochterski, P.Y. Ayala, K. Morokuma, A. Voth, P. Salvador, J.J. Dannenberg, V.G. Zakrzewski, S. Dapprich, A.D. Daniels, M.C. Strain, O. Farkas, D.K. Malick, A.D. Rabuck, K. Raghavachari, J.B. Foresman, J.V. Ortiz, Q. Cui, A.G. Baboul, S. Clifford, J. Cioslowski, B.B. Stefanov, G. Liu, A. Liashenko, P. Piskorz, I. Komaromi, R.L. Martin, D.J. Fox, T. Keith, M.A. Al-Aham, C.Y. Peng, A. Nanayakkara, M. Challacombe, P.M.W. Gill, B. Johnson, W. Chen, M.W. Wong, C. Gonzalez and J.A. Pople, Gaussian 03, Revision D.1, Gaussian, Inc., Pitttsburgh, PA, (2005).

11. C.L. Waller and J.D. McKinney, Chem. Res. Toxicol., 8, 847 (1995).

12. J.D. McKinney and L.G. Pedersen, J. Biochem., 240, 621 (1986).

13. R.G. Parr and Z. Zhou, Acc. Chem. Res., 26, 256 (1993).

14. R.G. Pearson, Acc. Chem. Res., 26, 250 (1993).

15. N.P. Barua, I. Sarmah, R. Hussain and C. Deka, Chem. Biol. Drug Des., 79, 553 (2012). 